\title{
Recent progress in hypernuclear physics
}

\author{
E. Hiyama ${ }^{1, a}$
}

Nishina Center for Accelerator-Based Science, Institute for Physical and Chemical Research (RIKEN), Wako, Saitama, 351-0198, Japan

\begin{abstract}
Hypernuclear physics has become very exciting owing to new epoch-making experimental data. The recent progress in theoretical and experimental studies of hypernuclei and discussion about the future development in this filed are done.
\end{abstract}

\section{Introduction}

One of the main goals in hypernuclear physics is to understand the baryon-baryon interaction. The baryonbaryon interaction is fundamental and important for the study of nuclear physics. In order to understand the baryon-baryon interaction, two-body scattering experiment is the most useful. For this purpose, many $N N$ scattering experiments have been done and the total number of $N N$ data are more than 4,000. However, due to the difficulty of performing two-body hyp$\operatorname{eron}(Y)$-nucleon $(N)$ and hyperon $(Y)$-hyperon $(Y)$ scattering experiments, the total number of $Y N$ scattering data are very limited. Namely, the number of differential cross section are only about 40 and there is no $Y Y$ scattering data. Then, $Y N$ and $Y Y$ potential models so far proposed have large ambiguity.

Therefore, as a substitute for the two-body limited $Y N$ and non-existent $Y Y$ scattering data, the systematic investigation of light hypernuclear structure is essential. Strategy to extract useful information about $Y N$ and $Y Y$ interactions from study of light hypernuclear structure is as follows (cf. Fig. 1):

i) Firstly, we have candidate $Y N$ and $Y Y$ interactions which are based on the meson

theory and the constituent quark model.

ii) Secondly, we have hypernuclear spectroscopy experiments performed in order to

provide information about the $Y N$ and $Y Y$ interactions. However, these experiments

do not directly give any information about the interactions.

iii) Therefore, using the interactions in i), accurate calculation of hypernuclear struc-

ture is performed. The calculated result is compared with the experimental data.

iv) From this comparison, suggestions to improve the interactions are proposed.

\footnotetext{
${ }^{a}$ e-mail: hiyama@riken.jp
}

Within the programs among i) to iv), the author's role is to contribute to iii) and iv) using an accurate three- and four-body calculational method developed by the author and her collaborators (cf. the next section).

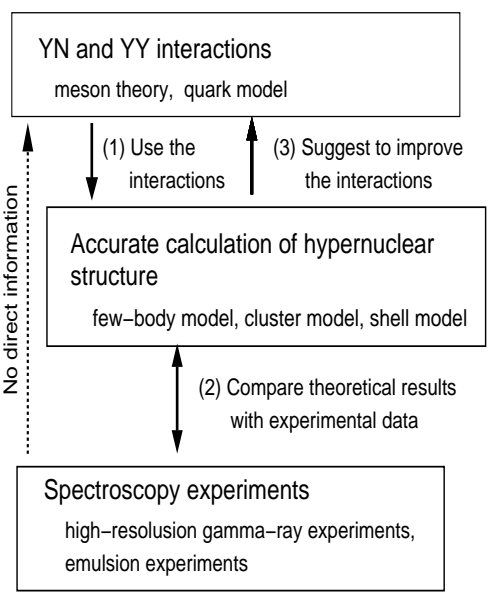

Fig. 1. Strategy for extracting information about $Y N$ and $Y Y$ interactions from the study of the structure of light hypernuclei

\section{$2 S=-1$ hypernuclei and $Y N$ interaction}

On the basis of the strategy mentioned in Sec. 1, we have obtained information about the spin-spin, spinorbit and tensor term of $Y N$ interaction from the study of $S=-1$ hypernuclei. As an example, the case of the study of $Y N$ spin-orbit force is explained.

In the $Y N$ interaction, there are two kinds of $L S$ forces, symmetric $L S$ force $(S L S)$ and antisymmetric $L S(A L S)$ force, defined by

$$
\begin{aligned}
& V_{S L S}=\mathbf{L} \cdot\left(\mathbf{s}_{\mathbf{\Lambda}}+\mathbf{s}_{\mathbf{N}}\right) v_{S L S}(r), \\
& V_{A L S}=\mathbf{L} \cdot\left(\mathbf{s}_{\boldsymbol{\Lambda}}-\mathbf{s}_{\mathbf{N}}\right) v_{A L S}(r),
\end{aligned}
$$


where $\mathbf{s}_{\Lambda}$ and $\mathbf{s}_{N}$ are spins of $\Lambda$ and $N$, respectively. The $A L S$ force vanishes in conventional nuclei because of the Pauli principle. On the other hand, the $A L S$ force is produced in hypernuclei since no Pauli principle works between $\Lambda$ and $N$.

Historically, it is well known that the $A L S$ force differs between meson theory and the constituent quark model [9]. For instance, the quark model of the KyotoNiigata group [10] predicts that the strength of the $A L S$ amounts to approximately $85 \%$ of that of the $S L S$ with the opposite sign. On the other hand, the meson based interaction of the Nijmegen group [11, 12 ] generates much smaller strength in the $A L S$, some $20-40 \%$ of that of the $S L S$ with the opposite sign. It is important to extract information about these $L S$ forces from the study of the structure of $\Lambda$ hypernuclei.

For the study of spin-orbit force, ${ }_{\Lambda}^{9} \mathrm{Be}$ and ${ }_{\Lambda}^{13} \mathrm{C}$ are very useful. Recently, in high-resolution $\gamma$-ray experiments, BNL-E930 [13] and BNL-E929 [14], the spinorbit splitting energies of ${ }_{\Lambda}^{9} \mathrm{Be}$ and ${ }_{\Lambda}^{13} \mathrm{C}$ were measured. Namely, the one (E930) observed $\gamma$ rays from the decay of the $5 / 2_{1}^{+}$and the $3 / 2_{1}^{+}$states to the $1 / 2_{1}^{+}$ ground state in ${ }_{1}^{9} \mathrm{Be}$, and the other (E929) measured those from the $3 / 2_{1}^{-}$and $1 / 2_{1}^{-}$states to the $1 / 2_{1}^{+}$ ground state in ${ }_{\Lambda}^{13} \mathrm{C}$.

Before the measurements, we predicted those energy splittings in [15]. we took an $\alpha+\alpha+\Lambda$ three-body model for ${ }_{\Lambda}^{9} \mathrm{Be}$ and an $\alpha+\alpha+\alpha+\Lambda$ four-body calculations for ${ }_{\Lambda}^{13} \mathrm{C}$. We employed two types of the $Y N$ spin-orbit force, namely, the Nijmegen meson-theory based $Y N$ interaction and the Kyoto-Niigata group's quark based $Y N$ interaction mentioned above. The predicted energy splittings of ${ }_{\Lambda}^{9} \mathrm{Be}$ and ${ }_{\Lambda}^{13} \mathrm{C}$ are listed in the second and third columns of Table 1 . In both nuclei, the splittings given by using the quark based $L S$ force is significantly smaller than those by using the meson based $L S$ force.

Recently, experimental data for these energy splittings of ${ }_{\Lambda}^{9} \mathrm{Be}$ [13] and ${ }_{\Lambda}^{13} \mathrm{C}$ [14] have been reported to be $43 \pm 5 \mathrm{keV}$ and $152 \pm 54 \pm 36 \mathrm{keV}$, respectively as shown in Table 1 . We see that the predicted energy splitting using the quark-model based spin-orbit force can explain both data. On the other hand, the predictions using the meson theory based one are much larger than the data.

The reason why meson theory based $Y N$ interaction proposed large spin-orbit splitting in the case of ${ }_{\Lambda}^{9} \mathrm{Be}$ is as follows: Using the $S L S$ force only, the splitting energy is $140-250 \mathrm{keV}$ depending on the five models in the $Y N$ interaction: it is not so small value. When the $A L S$ force is included, the $A L S$ with the opposite sign of the $S L S$ reduces this splitting. But, the strength of the $A L S$ in the case of Nijmegen model, $20-40 \%$ of the $S L S$ as mentioned before, is not enough to reproduce the observed data. On the other hand, in the quark model, the $A L S$ is strong enough to reproduce the data. Therefore, we suggested that there are two paths to improve the meson based model; one is to reduce the $S L S$ strength and the other is to enhance the $A L S$ strength so as to reproduce the observed spin-orbit splittings in ${ }_{\Lambda}^{9} \mathrm{Be}$ and ${ }_{\Lambda}^{13} \mathrm{C}$.

Recently, a new $Y N$ interaction based on meson theory was proposed by the Nijmegen group (ESC06) [16]; they proposed a reduced strength of the $S L S$. Using this potential, we obtained, as shown in Table 2 , the energy splitting in ${ }_{\Lambda}^{9}$ Be to be $98 \mathrm{keV}$ in the case of the $S L S$ only and $39 \mathrm{keV}$ with including the $A L S$, which is in good agreement with the data.

To summarize with referring to the numbers in the parentheses in the strategy diagram of Fig. 1, (1) we used two types of the $Y N$ spin-orbit models, the Nijmegen model and the Kyoto-Niigata model and calculated the energy splittings of ${ }_{\Lambda}^{9} \mathrm{Be}$ and ${ }_{\Lambda}^{13} \mathrm{C}$. (2) We then compared our results with the experimental data. (3) We suggested improving the strength of the $L S$ force. After that, the Nijmegen group proposed a new potential version ESC06. Using this potential, we calculated the energy splitting, and we compared them with the experimental data. Then, the calculated results were in good agreements with the experimental data. Since 1998 , we have many $\gamma$-ray spectroscopic data $[17,18]$. By the combined analysis of experiments and theoretical calculations, we succeeding in extracting information about the spin-spin, spin-orbit and tensor terms of $\Lambda N$ interaction.

\section{$3 S=-2$ hypernuclei and $Y Y$ interaction}

It is interesting to investigate the structure of the multi-strangeness system when one or more $\Lambda$ s are added to a $S=-1$ nucleus. It is conjectured that extreme limit, which includes many $\Lambda$ s (and other hyperons) in nuclear matter is the core of a neutron star. In this meaning, the sector of $S=-2$ nuclei, double $\Lambda$ hypernuclei and $\Xi$ hypernuclei, is just the entrance to the multi-strangeness world. However, we have hardly any knowledge of the $Y Y$ interaction because there exist no $Y Y$ scattering data. Then, in order to understand the $Y Y$ interaction, it is crucial to study the structure of double $\Lambda$ hypernuclei and $\Xi$ hypernuclei. The equation of state with the strangeness degree of freedom is a crucial component in understanding neutron stars.

Recently, the epoch-making data has been reported by the KEK-E373 experiment. Namely, the double $\Lambda$ hypernucleus ${ }_{\Lambda \Lambda}^{6}$ He was observed [19]. This observation was called NAGARA event. The formation of ${ }_{\Lambda \Lambda}^{6} \mathrm{He}$ was uniquely identified by the observation of sequential weak decays, and the precise experimental value of the $2 \Lambda$ binding (separation) energy, $B_{\Lambda \Lambda}=$ $7.25 \pm 0.19_{-0.11}^{+0.18} \mathrm{MeV}$, was obtained.

Following the strategy mentioned in Sec. 1, we studied double $\Lambda$ hypernuclei with $A=6-10$ [20]. Firstly, (1) we employed the $\Lambda \Lambda$ interaction of Nijmegen model $\mathrm{D}$ and performed an $\alpha+\Lambda+\Lambda$ three-body calculation for ${ }_{\Lambda \Lambda}^{6}$ He. (2) By comparing the theoretical 


\section{$19^{\text {th }}$ International IUPAP Conference on Few-Body Problems in Physics}

Table 1. Spin-orbit splitting energy in in ${ }_{\Lambda}^{9} \mathrm{Be}$ and ${ }_{\Lambda}^{13} \mathrm{C}$. Calculated values are given by Hiyama et al. [15] using the meson-theory based $\Lambda N$ spin-orbit force $[11,12]$ and the quark-model based one [10]. Experimental values are taken from [13] for ${ }_{\Lambda}^{9} \mathrm{Be}$ and from [14] for ${ }_{\Lambda}^{13} \mathrm{C}$.

\begin{tabular}{lcccc}
\hline \hline & splitting & $\begin{array}{c}\text { CAL(meson theory) } \\
(\mathrm{keV})\end{array}$ & $\begin{array}{c}\mathrm{CAL}(\text { quark model }) \\
(\mathrm{keV})\end{array}$ & $\begin{array}{c}\text { EXP } \\
(\mathrm{keV})\end{array}$ \\
\hline${ }_{\Lambda}^{9} \mathrm{Be}:$ & $E\left(5 / 2_{1}^{+}-3 / 2_{1}^{+}\right)$ & $80-200$ & $35-40$ & $43 \pm 5$ \\
${ }_{\Lambda}^{13} \mathrm{C}:$ & $E\left(3 / 2_{1}^{-}-1 / 2_{1}^{-}\right)$ & $390-960$ & $150-200$ & $150 \pm 54 \pm 36$ \\
\hline \hline
\end{tabular}

Table 2. Calculated spin-orbit splitting energy in in ${ }_{\Lambda}^{9}$ Be using an improved meson theory based $L S$ force (ESC06) [16]. Contribution of $S L S$ is shown in comparison with that of $S L S+A L S$. CAL(meson theory) is the same as in Table 1.

\begin{tabular}{|c|c|c|c|c|c|}
\hline \multirow{2}{*}{$\begin{array}{l}\text { splitting } \\
(\mathrm{keV})\end{array}$} & \multicolumn{2}{|c|}{ CAL(meson theory) } & \multicolumn{2}{|r|}{ CAL(ESC06) } & \multirow{2}{*}{$\begin{array}{l}\text { EXP } \\
(\mathrm{keV})\end{array}$} \\
\hline & $S L S$ & $S L S+A L S$ & $S L S$ & $S L S+A L S$ & \\
\hline${ }_{\Lambda}^{9} \mathrm{Be}: E\left(5 / 2_{1}^{+}-3 / 2_{1}^{+}\right)$ & $140-250$ & $80-200$ & 98 & 39 & $43 \pm 5$ \\
\hline
\end{tabular}

result with the experimental data of the binding energy of ${ }_{\Lambda \Lambda}^{6} \mathrm{He}$, (3) we suggested reducing the strength of ${ }^{1} S_{0}$ term of the $\Lambda \Lambda$ interaction by half to reproduce the data. Again, (2) using the improved potential, we predicted energy spectra of new double $\Lambda$ hypernuclei with $A=7-10$ [20], which is discussed below.

In fact, it is planned at J-PARC to produce many double $\Lambda$ hypernuclei by emulsion experiment [21]. However, it will be difficult to determine spin-parities and to know whether the observed state is the ground state or an excited state. Therefore, it will be necessary to compare the data with any theoretical study for the identification of the state. The author's role is to contribute to the theoretical calculation using fewbody techniques.

A successful example to determine spin-parity of double $\Lambda$ hypernuclei is the case of ${ }_{\Lambda \Lambda}^{10}$ Be. There was one more event found in the E373 experiment named the 'Demachi-Yanagi' event $[22,23]$. The most probable interpretation of this event is the production of a bound state of ${ }_{\Lambda \Lambda}^{10}$ Be having $B_{\Lambda \Lambda}^{\exp }=12.33_{-0.21}^{+0.35} \mathrm{MeV}$. But the experiment could not determine whether this state was the ground state or any excited state. In order to determine this, our calculation [20] mentioned above was useful as following: We studied ${ }_{\Lambda \Lambda}^{10} \mathrm{Be}$ by employing an $\alpha+\alpha+\Lambda+\Lambda$ four-body model. The $\Lambda \Lambda$ interaction is the one improved from the Nijmegen Model $\mathrm{D}$ as mentioned above. The $\Lambda \Lambda, \alpha \Lambda$ and $\alpha \alpha$ interactions were chosen so as to reproduce the binding energies of all the subsystems, ${ }_{\Lambda \Lambda}^{6} \mathrm{He},{ }_{\Lambda}^{5} \mathrm{He}$, ${ }^{8} \mathrm{Be}$ and ${ }_{A}^{9} \mathrm{Be}$. As shown in Fig. 2, it is striking that our calculated value of $B_{\Lambda \Lambda}\left({ }_{\Lambda \Lambda}^{10} \mathrm{Be}\left(2^{+}\right)\right)$is $12.28 \mathrm{MeV}$ that agrees with the experimental data. Therefore, the Demachi-Yanagi event can be interpreted most probably as the observation of the $2^{+}$excited state in ${ }_{\Lambda \Lambda}^{10} \mathrm{Be}$.

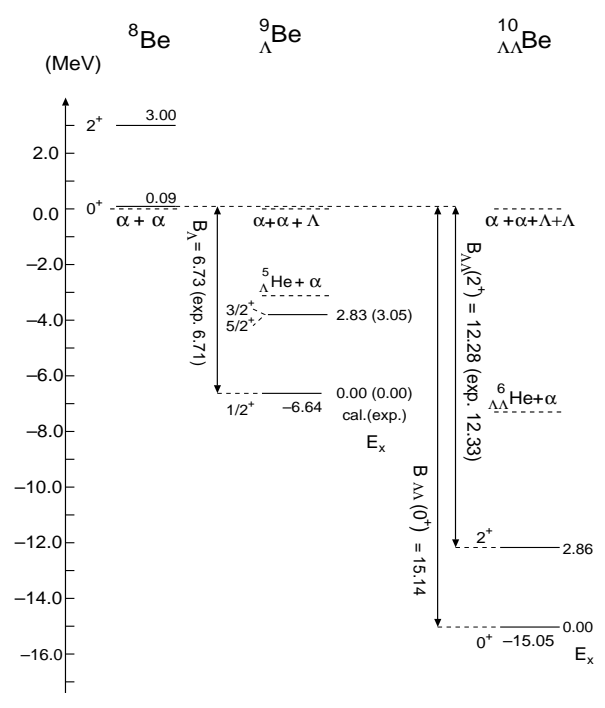

Fig. 2. Calculated energy levels of ${ }^{8} \mathrm{Be},{ }_{\Lambda}^{9} \mathrm{Be}$ and ${ }_{\Lambda \Lambda}^{10} \mathrm{Be}$ on the basis of the $\alpha+\alpha, \alpha+\alpha+\Lambda$, and $\alpha+\alpha+\Lambda+$ $\Lambda$ models, respectively. The level energies are measured from the particle breakup thresholds or are given by the excitation energies $E_{\mathrm{x}}$. The calculated $2^{+}$state of ${ }_{41}^{10} \mathrm{Be}$ explains the Demachi-Yanagi event. This figures is taken from $[20]$

In this way, we succeeded in interpreting the spinparity of ${ }_{\Lambda \Lambda}^{10} \mathrm{Be}$ by comparing the experimental data and our theoretical calculation. Therefore, our fourbody calculation is considered to have a predictive power. Hoping to observe new double $\Lambda$ hypernuclei in future experiments, we have predicted, as shown in Fig. 3, level structure of double $\Lambda$ hypernuclei with 
EPJ Web of Conferences

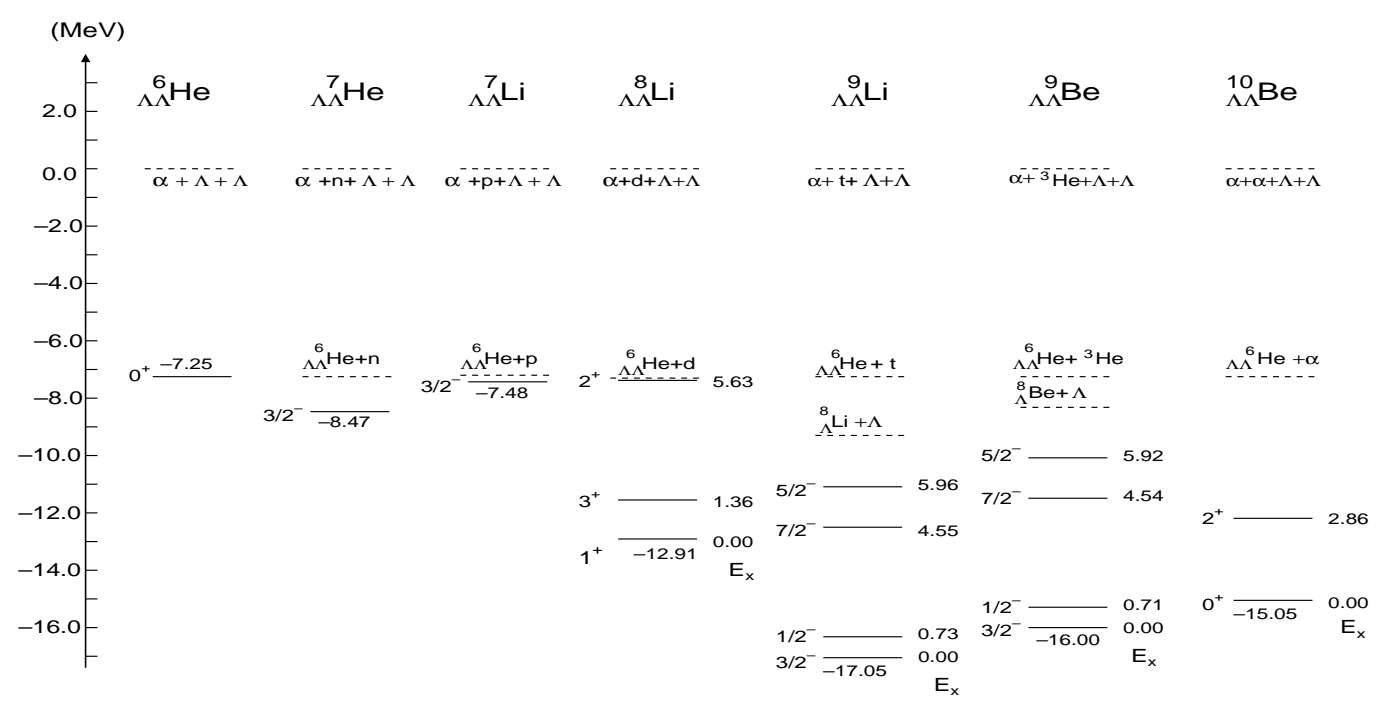

Fig. 3. Energy levels of double- $\Lambda$ hypernuclei, ${ }_{\Lambda \Lambda}^{6} \mathrm{He},{ }_{\Lambda \Lambda}^{7} \mathrm{He},{ }_{\Lambda \Lambda}^{7} \mathrm{Li},{ }_{\Lambda \Lambda}^{8} \mathrm{Li},{ }_{\Lambda \Lambda}^{9} \mathrm{Li},{ }_{\Lambda \Lambda}^{9}$ be and ${ }_{\Lambda \Lambda}^{10}$ Be calculated using the $\alpha+x+\Lambda+\Lambda$ model with $x=0, n, p, d, t,{ }^{3}$ He and $\alpha$, respectively. This figure is taken from [20].

$A=7-9$ taking the framework of the $\alpha+x+\Lambda+\Lambda$ models with $x=n, p, d, t$ and ${ }^{3} \mathrm{He}[20]$.

\section{Observation of Hida event}

In the KEK-E373 experiments, they have observed the two events of double $\Lambda$ hypernuclei, named as Nagara, Demachi-Yanagi $[19,22,23]$. Recently, since the mass of $\Xi^{-}$has been modified by $0.4 \mathrm{MeV}$ in the PDB, they have re-analyzed them to be $B_{\Lambda \Lambda}=6.91 \pm 0.16$ $\mathrm{MeV}$ for ${ }_{\Lambda \Lambda}^{6}$ He and to be $B_{\Lambda \Lambda}=11.90 \pm 0.13 \mathrm{MeV}$ for ${ }_{\Lambda \Lambda}^{10} \mathrm{Be}$. Also, in the KEK-E373 experiments, they observed one more event, Hida event [24]. This observation is for ${ }_{\Lambda \Lambda}^{11} \mathrm{Be}$ or ${ }_{\Lambda \Lambda}^{12} \mathrm{Be}$. The observed $B_{\Lambda \Lambda}$ for ${ }_{\Lambda \Lambda}^{11} \mathrm{Be}$ is $20.49 \pm 1.15 \mathrm{MeV}$ and $B_{\Lambda \Lambda}$ for ${ }_{\Lambda \Lambda}^{12} \mathrm{Be}$ is $22.06 \pm 1.15$ $\mathrm{MeV}$ [24]. Then, we have two important issues: (1) Can we reproduce the revised Deamchi-Yanagi event using $\Lambda \Lambda$ interaction which reproduce the revised NAGARA event? (2) Is the Hida event the observation of ${ }_{\Lambda \Lambda}^{11} \mathrm{Be}$ or ${ }_{\Lambda \Lambda}^{12} \mathrm{Be}$ ?

In order to answer issue (1), we perform four-body calculation of $\alpha \alpha \Lambda \Lambda$ model for ${ }_{\Lambda \Lambda}^{10} \mathrm{Be}$. The employed $\Lambda \Lambda$ interaction is adjusted so as to reproduce the observed $B_{\Lambda \Lambda}=6.91 \pm 0.16$ of ${ }_{\Lambda \Lambda}^{6} \mathrm{He}$. Calculated $B_{\Lambda \Lambda}$ of $2^{+}$state for ${ }_{\Lambda \Lambda}^{10} \mathrm{Be}$ is $11.83 \mathrm{MeV}$ which is in good agreement with the revised data. Then, we can still interpret the Demachi-Yanagi event as a observation of the $2^{+}$excited state. Next, we discuss the Hida event. We assume Hida event as ${ }_{\Lambda \Lambda}^{11} \mathrm{Be}$ and calculate the $B_{\Lambda \Lambda}$ with $\alpha \alpha n \Lambda \Lambda$ five-body problem. This five-body calculation is numerically difficult since we have three kinds of particles such as $\alpha, \Lambda$ and neutron, and we have five different kinds of interactions such as $\Lambda \Lambda, \Lambda n, \Lambda \alpha, n \alpha$ and $\alpha \alpha$, and we have Pauli principle between $\alpha$ and $\alpha$, and between $\alpha$ and neutron. Recently, we succeeded in performing this calculation.
In the present $\alpha+\alpha+n+\Lambda+\Lambda$ five-body model for ${ }_{\Lambda \Lambda}^{11} \mathrm{Be}$, it is absolutely necessary that all sub-cluster systems composed of two $\alpha$ 's, a neutron and two $\Lambda$ 's are described reasonably with the interactions among these units. In our previous work [20], our interactions, which include the $\alpha \alpha, \alpha n, \alpha \Lambda, \Lambda n$ and $\Lambda \Lambda$ interactions, were determined so as to reproduce reasonably well the following observed quantities: (i) Energies of the low-lying states and scattering phase shifts in the $\alpha+n$ and $\alpha+\alpha$ systems, (ii) $\Lambda$-binding energies $B_{\Lambda}$ in ${ }_{\Lambda}^{5} \mathrm{He}$ $(=\alpha+\Lambda),{ }_{\Lambda}^{6} \mathrm{He}(=\alpha+\Lambda+n)$ and ${ }_{\Lambda}^{9} \mathrm{Be}(=\alpha+\alpha+\Lambda)$, (iii) double- $\Lambda$ binding energies $B_{\Lambda \Lambda}$ in ${ }_{\Lambda \Lambda}^{6} \mathrm{He}(=\alpha+$ $\Lambda+\Lambda)$, the Nagara event. Then, as mentioned above, the Demachi-Yanagi event for ${ }_{\Lambda \Lambda}^{10} \mathrm{Be}(=\alpha+\alpha+\Lambda+$ A) was simultaneously reproduced with no additional adjustable parameter.

In the present work, we employ the same interactions of Ref.[20] so that those severe constraints are also successfully met in our two-, three- and four-body subsystems. But, as for the present core nucleus ${ }^{9} \mathrm{Be}$ $(=\alpha+\alpha+n)$, which does not belong to the subsystems studied previously, use of the interactions that explain well the property of the $\alpha \alpha$ and $\alpha n$ subsystems do not well reproduce the energies of the low-lying states of ${ }^{9}$ Be measured from the $\alpha+\alpha+n$ threshold (the same property of the calculated result was reported in another microscopic $\alpha+\alpha+n$ cluster-model study [25]). Therefore, we additionally introduce a phenomenological $\alpha \alpha n$ three-body force with a Gaussian shape, $v_{0} e^{-\left(r_{\alpha-\alpha} / r_{0}\right)^{2}-\left(R_{\alpha \alpha-n} / R_{0}\right)^{2}}$, having $r_{0}=$ $3.6 \mathrm{fm}, R_{0}=2.0 \mathrm{fm}$ and $v_{0}=-9.7 \mathrm{MeV}(+13.0 \mathrm{MeV})$ for the negative-parity (positive-parity) state; we thus reproduce well the observed energies of the $3 / 2_{1}^{-}, 5 / 2_{1}^{-}$, $1 / 2_{1}^{-}$and $1 / 2_{1}^{+}$states of ${ }^{9} \mathrm{Be}$. The calculated $B_{\Lambda \Lambda}$ of the ground state in ${ }_{\Lambda \Lambda}^{11} \mathrm{Be}$ is $18.23 \mathrm{MeV}$ which is not contradict with the observed value of Hida event, $B_{\Lambda \Lambda}^{\exp }=20.49 \pm 1.15 \mathrm{MeV}[24]$ within the two $\sigma$ er- 


\section{$19^{\text {th }}$ International IUPAP Conference on Few-Body Problems in Physics}

ror bar. For the confirmation of Hida event, we expect to have more precise data at J-PARC facility in the future.

\section{$5 \Xi$ hypernuclei}

For the study of $\Xi N$ interaction, it is important to study the structure of $\Xi$ hypernuclei. Our intention in this section is to investigate the possible existence of $\Xi$ hypernuclei and to explore the properties of the underlying $\Xi N$ interactions. Identification of $\Xi$ hypernuclei in coming experiments at J-PARC will contribute significantly to understanding nuclear structure and interactions in $S=-2$ systems, which can lead to an entrance into the world of multi-strangeness. In order to encourage new experiments seeking $\Xi$ hypernuclei, it is essential to make a detailed theoretical investigation of the possible existence of bound states, despite some uncertainty in contemporary $\Xi N$ interaction models.

We investigate here the binding energies and structure of $\Xi$ hypernuclei produced by $\left(K^{-}, K^{+}\right)$reactions on light targets on the basis of microscopic cluster models. One of the primary issues is how to choose the $\Xi N$ interaction. Although there are no definitive data for any $\Xi$ hypernucleus at present, a few experimental data indicate that $\Xi$-nucleus interactions are attractive. One example is the observed spectrum of the $\left(K^{-}, K^{+}\right)$reaction on a ${ }^{12} \mathrm{C}$ target, where the cross sections for $\Xi^{-}$production in the threshold region can be interpreted by assuming a $\Xi$-nucleus WoodSaxon (WS) potential with a depth of $\sim 14 \mathrm{MeV}[26]$. Other indications of attractive $\Xi$-nucleus interactions are given by certain emulsion data, the events for twin$\Lambda$ hypernuclei, where the initial $\Xi^{-}$energies were determined by the identification of all fragments after the $\Xi^{-} p-\Lambda \Lambda$ conversion in nuclei. The inferred $\Xi^{-}$ binding energies are substantially larger than those obtained using only the Coulomb interaction [27]. When these $\Xi^{-}$states are assumed to be $1 p$ states, the WS potentials obtained from the binding energies are similar to the one above. These data suggest that the average $\Xi N$ interaction should be attractive, which we utilize to select the appropriate interaction models. In this work we adopt two types of $\Xi N$ interactions, the Nijmegen Hard-Core model D (ND) [28] and the Extended Soft-Core model (ESC04) $[29,30]$.

The structure of light $p$-shell nuclei can be reasonably described in terms of cluster models composed of two- or three-body subunits. Here, we model the possible $\Xi^{-}$hypernuclei produced by $\left(K^{-}, K^{+}\right)$reactions on available light $p$-shell targets as four-body cluster structures: The possible targets ${ }^{12} \mathrm{C},{ }^{11} \mathrm{~B},{ }^{10} \mathrm{~B},{ }^{9} \mathrm{Be}$ and ${ }^{7} \mathrm{Li}$ naturally lead to such cluster configurations as $\alpha \alpha t \Xi^{-}\left(\Xi^{-} \mathrm{Be}\right), \alpha \alpha 2 n \Xi^{-}\left(\Xi_{\Xi}^{11} \mathrm{Li}\right), \alpha \alpha n \Xi^{-}\left(\Xi^{-} \mathrm{Li}\right), \alpha t n \Xi^{-}$ and $\alpha n n \Xi^{-}\left(\Xi^{7} \mathrm{H}\right)$, respectively, by conversion of a proton into a $\Xi^{-}$. (In our model calculations, the $\alpha \Xi^{-}$ potential is generated from a G-matrix $\Xi N$ interaction via a folding procedure.) In the case of lighter targets, ${ }^{6} \mathrm{Li},{ }^{4} \mathrm{He},{ }^{3} \mathrm{He}$ and $d$, the $\Xi^{-}$-hypernuclear states are composed of $\alpha n \Xi^{-}, p n n \Xi^{-}\left(t \Xi^{-}\right), p n \Xi^{-}$ and $n \Xi^{-}$configurations, respectively. However, these systems are not expected to support bound states, considering the weakly attractive nature of the $\Xi N$ interactions suggested so far, except for Coulomb-bound (atomic) states. Then, among the above $\Xi^{-}$hypernuclei, $\Xi^{7} \mathrm{H}\left(\alpha n n \Xi^{-}\right)$is expected to be the lightest $\Xi^{-}$ bound system. Thus, possible $\Xi^{-}$hypernuclear states to be investigated lie in the light $p$-shell region and may be considered to have basically a four-body cluster structure.

In this work, we adopt two-types of $G$-matrix $\Xi N$ potentials derived from the Nijmegen hard-core model $\mathrm{D}(\mathrm{ND})[28]$ and the extended soft-core model (ESC04) $[29,30]$. The detailed potential parameters in these $G$-matrix $\Xi N$ interaction are listed in Table II in Ref.[31]. It is should be noted here that the even-state interaction of ESC04 is more attractive than that of $\mathrm{ND}$, while the odd-state interaction of ND is more attractive than ESC04. The calculated energies of $\alpha \Xi^{-}$ systems which is dominated by even-state spin- and isospin- independent part, are $-1.36 \mathrm{MeV}$ for ESC04 and $-0.57 \mathrm{MeV}$ for ND, respectively. And the calculated energies of $\alpha \alpha \Xi^{-}$systems are $-4.81 \mathrm{MeV}$ for ESC04 and $-2.87 \mathrm{MeV}$ for ND. In the $\alpha \alpha \Xi^{-}$system, odd state spin-and isospin-independent term contributes significantly. Thus, we can say that observations of $\alpha \Xi^{-}$and $\alpha \alpha \Xi^{-}$systems certainly provide information about spin-independent parts of the $\Xi N$ interactions.

$\Xi^{7}-H(E S C)$

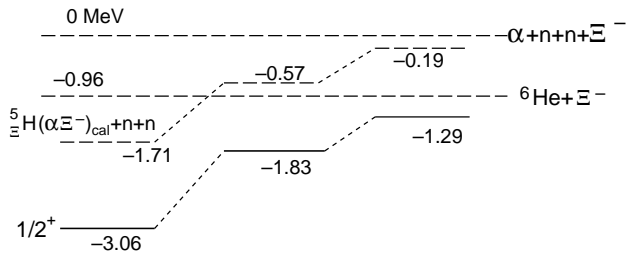

$\begin{array}{lll}\text { (i) } k_{F}=0.9 & \text { (ii) } k_{F}=1.055 & \text { (iii) } k_{F}=1.3\end{array}$

${ }_{\Xi}^{10} \operatorname{Li}(E S C)$

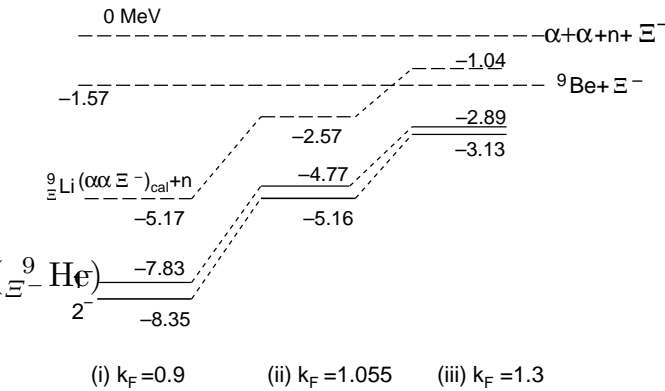

Fig. 4. Calculated energy levels of (a) $\Xi_{\Xi^{-}}^{7} \mathrm{H}$ and (b) $\Xi_{\Xi^{-}}^{10} \mathrm{Li}$ for three $k_{\mathrm{F}}$ values using ESC. 
In reality, however, there are no corresponding nuclear targets to produce the above systems by the $\left(K^{-}, K^{+}\right)$reaction. As their actual substitutes, in the following, we investigate the structures of ${ }_{\Xi^{-}}^{7} \mathrm{H}\left(\alpha n n \Xi^{-}\right)$and $\stackrel{\Xi}{\Xi}-^{10} \operatorname{Li}\left(\alpha \alpha n \Xi^{-}\right)$having additional neutron(s) and propose to perform the ${ }^{7} \operatorname{Li}\left(K^{-}, K^{+}\right)$ and ${ }^{10} \mathrm{~B}\left(K^{-}, K^{+}\right)$reaction experiments with available targets. The calculated energies in the $1 / 2^{+}$ground state for $\Xi^{7} \mathrm{H}\left(\alpha n n \Xi^{-}\right)$and $1^{-}$and $2^{-}$state for $\Xi^{-} \mathrm{Li}$ using ESC are demonstrated in Fig.3 as a function of $k_{F}$.

In $\mathrm{A}=7$ hypernucleus, the four-body calculation predicts the existence of nuclear bound states in ESC case at reasonable $k_{\mathrm{F}}$ values of around $0.9 \mathrm{fm}^{-1}$. It is interesting to note that the addition of two neutrons to the $\alpha \Xi^{-}$system gives rise to about $1.3(2.0) \mathrm{MeV}$ more binding. The same tendency is seen in ND. This means that an experimental finding of $\mathrm{a}_{\Xi_{-}}^{7} \mathrm{H}$ bound state indicates the existence of an $\alpha \Xi^{-}$bound state in which the even-state spin- and iso-spin independent part of the $\Xi N$ substantially attractive. This statement is almost independent on the interaction model.

In $\mathrm{A}=10$ hypernucleus, we have obtained the nuclear $\Xi^{-}$bound states as a result of careful four-body calculations with $k_{\mathrm{F}} \sim 1.0 \mathrm{fm}^{-1}$. Furthermore, we have similar binding energies of the $J=2^{-}$state for both the ESC and ND interactions. Then, to produce ${ }_{\Xi}^{10} \mathrm{Li}$, we propose to perform the ${ }^{10} \mathrm{~B}\left(K^{-}, K^{+}\right)$reaction experiment at J-PARC in addition to that with a ${ }^{12} \mathrm{C}$ target. We say that the $\alpha \alpha n \Xi^{-}\left({ }_{\Xi}^{10} \mathrm{Li}\right)$ system produced by the $\left(K^{-}, K^{+}\right)$reaction on ${ }^{10} \mathrm{~B}$ is suitable to investigate $\alpha \Xi^{-}$interaction, namely the spin- and iso-spin independent terms of even and odd-state $\Xi N$ interactions.

\section{Acknowledgement}

The numerical calculations were done on HITACHI SR11000 at KEK.

\section{References}

1. M. Kamimura, Phys. Rev. A38 (1988) 621.

2. E. Hiyama, Y. Kino, M. Kamimura, Prog. Part. Nucl. Phys. 51 (2003) 223.

3. S. Eidelman et al., Phys. Lett. B592 (2004) 1.

4. Y. Kino, M. Kamimura, H. Kudo, Proc. Int. Conf. on Low Energy Antiproton Physics, Yokohama, 2003, Nucl. Instrum. Methods Phys. Res. B214 (2004) 84

5. M. Hori et al., Phys. Rev. Lett. 91 (2003) 123401.

6. H. Kamada et al., Phys. Rev. C64 (2001) 044001.

7. E. Hiyama, B.F. Gibson and M. Kamimura, Phys. Rev. C70, (2004) 031001(R).

8. E. Hiyama, M. Kamimura, A. Hosaka, H. Toki and M. Yahiro, Phys. Lett. B633 (2006) 237.

9. O. Morimatsu, S. Ohta, K. Shimizu and K. Yazaki, Nucl. Phys. A420(1984) 573.

10. Y. Fujiwara, C. Nakamoto and Y. Suzuki, Phys. Rev. C59 (1999) 21.
11. M.M. Nagels, T. A. Rijken and J. J. deSwart, Phys. Rev. D12 (1975) 744; 15 (1977) 2547; 20 (1979) 1633.

12. T. A. Rijken, V.G. Stoks and Y. Yamamoto, Phys. Rev. C59 (1999) 21.

13. H. Akikawa et al., Phys. Rev. Lett. 88 (2002) 82501;H. Tamura et al., Nucl. Phys. A754 (2005) 58c.

14. S. Ajimura et al., Phys. Rev. Lett. 86 (2001) 4225.

15. E. Hiyama, M. Kamimura, T. Motoba, T. Yamada and Y. Yamamoto, Phys. Rev. Lett. 85 (2000) 270.

16. T. A. Rijken, private comminucation (2006).

17. O. Hashimoto and H. Tamura, Prog. Part. Nucl. Phys. 57 (2006) 564.

18. H. Tamura, in Proceedings on Interanational Nuclear Physics Conference 2007, to be published.

19. H. Takahashi et al., Phys. Rev. Lett. 87 (2002) 212502.

20. E. Hiyama, M. Kamimura, T. Motoba, T. Yamada and Y. Yamamoto, Phys. Rev. C 66 (2002) 024007.

21. K. Imai, K. Nakazawa, H. Tamura et al., J-PARC proposal No.E07, 2006.

22. K. Ahn et al., In Hadron and Nuclei, edited by II-Tong Chen et al., AIP Conf. Proc. 594 (2001) 180 .

23. A. Ichikawa, Ph.D. thesis, Kyoto Universitym 2001.

24. K.Nakazawa et al., in this proceedings, and to be submitted to Phys. Rev. C.

25. K. Arai, P. Descouvemont, D. Baye, and W. N. Catford, Phys. Rev. C68, (2003) 014310.

26. P. Khaustov et al., Phys. Rev. C61, (2000) 054603.

27. S. Aoki et al., Prog. Theor. Phys. 89 (1993), 493.; S. Aoki et al., Phys. Lett. B355 (1995), 45.; Y. Yamamoto, Genshikaku Kenkyu 39 (1996), 23.

28. M. M. Nagels, T. A. Rijken, and J. J. deSwart, Phys. Rev. D15 (1977) 2547.

29. Th. A. Rijken and Y. Yamamoto, Phys. Rev. C73, 044008 (2006); [arXiv:nucl-th/0603042]

30. Th. A. Rijken and Y. Yamamoto, [arXiv:nuclth/0608074]

31. E. Hiyama, Y. Yamamoto, T. Motoba, Th. A. Rijken, and M. kamimura, Phys. Rev. C78 (2008) 054316 . 